\title{
Non-adiabatic transition probability dependence on conical intersection topography
}

João Pedro Malhado ${ }^{1}$ and James T. Hynes ${ }^{2,3}$

1) Department of Chemistry, Imperial College London, London $S W^{\eta} 2 A Z$, $U K$

2) École Normale Supérieure-PSL Research University, Chemistry Department, Sorbonne Universités-UPMC University Paris 06, CNRS UMR 8640 Pasteur, 24 rue Lhomond, 75005 Paris, France

${ }^{3)}$ Department of Chemistry and Biochemistry, University of Colorado, Boulder, CO 80309-0215, USA

(Dated: November 17, 2016)

We derive a closed form analytical expression for the non-adiabatic transition probability for a distribution of trajectories passing through a generic conical intersection (CI), based on the Landau-Zener equation for the non-adiabatic transition probability for a single straight-line trajectory in the CI's vicinity. We investigate the non-adiabatic transition probability's variation with topographical features and find, for the same crossing velocity, no intrinsic difference in efficiency at promoting nonadiabatic decay between peaked and sloped CIs, a result in contrast to the commonly held view. Any increased efficiency of peaked over sloped CIs are thus due to dynamical effects rather than to any increased transition probability of topographical origin. It is also shown that the transition probability depends in general on the direction of approach to the CI, and that the coordinates' reduced mass can affect the transition probability via its influence on the CI topography in mass-scaled coordinates. The resulting predictions compare well with surface hopping simulation results. 


\section{INTRODUCTION}

Conical intersections (CIs) have been known for many years ${ }^{1,2}$ but only in the last several decades has their central role been emphasized in photochemistry ${ }^{3-7}$, and for photochemical reaction events occurring on the femtosecond time scale in particular. Their important role in photochemistry of leading to the efficient and rapid transition from excited to ground electronic states has been compared to the role of transition states in thermal chemical reactions ${ }^{8-11}$. In contrast to the situation for thermal chemical reactions where powerful analytic theories such as transition state theory, and qualitative relations such as the Polanyi rules $^{12}$ systematize the rate of chemical reactions based the transition state properties, a comparable theoretical arsenal for processes involving CIs which would assist the rationalization of results of both experiment and increasingly sophisticated and difficult-to-interpret computer simulations has not yet emerged ${ }^{11,13}$. One important illustration of this lack is provided by the fact that while CI topography ${ }^{14}$ is often invoked to explain the outcome of processes occurring at $\mathrm{CIs}^{15-21}$, the questions of the CI topography effect on the probability or the rate of non-adiabatic transitions have been addressed in systematic fashion in only very few, mostly computational, studies ${ }^{22,23}$. To be sure, it is generally understood that sloped CIs are less efficient than peaked CIs driving the decay from the excited state ${ }^{14,16,20-22}$, since the topography of potential energy surfaces will drive the system's dynamics towards the degeneracy in the latter case, and away from it in the former case. A further question - and the one addressed in this work - is whether besides this more-or-less intuitive dynamical effect, there is an effect of the CI topography on the efficiency of the non-adiabatic process itself. Alternatively expressed, is there an effect of the CI topography on the probability of non-adiabatic transition?

In one of the first accounts concerning $\mathrm{CIs}^{2}$, Teller applied the Landau-Zener (LZ) equation $^{24-2728}$ to determine the probability of non-adiabatic decay in the vicinity of a circular vertical CI, a particular case of a peaked CI. This analytical approach of the LZ equation application to the CI problem was pursued and extended by Nikitin ${ }^{29-33}$, and later applied by the group of Lorquet in some interesting molecular scenarios ${ }^{34-36}$. These studies were also focussed on the vertical CI case and thus did not address the issues of varying CI topogra-

phy. In this article, we show that the Teller approach ${ }^{2,37}$ can be applied to an arbitrary CI, derive an analytical expression for the non-adiabatic decay probability in its vicinity, and 
assess how this probability varies with the CI topography.

The outline of the remainder of this article is as follows. In section II, we first derive a LZbased equation for the non-adiabatic transition probability for a single trajectory passing in the vicinity of a generic CI; on the basis of this equation, we derive an equation for the transition probability of a system described by a distribution of trajectories crossing the CI region. Section III compares the predictions of the derived results with numerical surface hopping simulations, illustrating how the transition probability varies with different topographical features of the CI. Section IV offers some concluding remarks.

\section{AN EXPRESSION FOR NON-ADIABATIC TRANSITION PROBABILITY AT A CI}

In the absence of spin-orbit coupling ${ }^{38}$, a CI corresponds to a hypersurface of dimension $N-2$, where $N$ is the number of the molecular system's internal coordinates, within which the energies of two electronic adiabatic states of the same spin multiplicity are degenerate (different conditions apply when more than 2 electronic states are degenerate ${ }^{39}$, but this case is not considered here). Such a hypersurface is called a CI seam. For any point on the seam, the degeneracy is lifted linearly on a plane spanned by two internal coordinates. This plane is termed the branching plane or the branching space, where the electronic energy surfaces of the two states — which in the Born-Oppenheimer approximation correspond to the potential energy surfaces for the nuclear motion - have the shape of a double cone. In the vicinity of the $\mathrm{CI}$ in which a linear expansion of the electronic energy surfaces is valid, these can generically be represented in cartesian form as ${ }^{14,40}$

$$
V_{ \pm}(x, y)=A_{x} x+A_{y} y \pm \sqrt{B_{x} x^{2}+B_{y} y^{2}}
$$

where $x$ and $y$ are two appropriately mass-scaled coordinates in the branching space with the origin at the CI point. For the purposes of this study, it proves more convenient to rewrite eq. (1) in polar form, with $\rho=\sqrt{x^{2}+y^{2}}$ and $\phi=\arctan (y / x)$

$$
V_{ \pm}(\rho, \phi)=F \rho\left(\tan \left(\alpha_{x}\right) \cos (\phi)+\tan \left(\alpha_{y}\right) \sin (\phi) \pm \sqrt{\cos ^{2}(\phi)+e \sin ^{2}(\phi)}\right)
$$

where $e$ is an eccentricity or ellipticity parameter measuring the elliptical character of the double cone surface (for $e=1$ the double cone is circular and has cylindrical symmetry), 

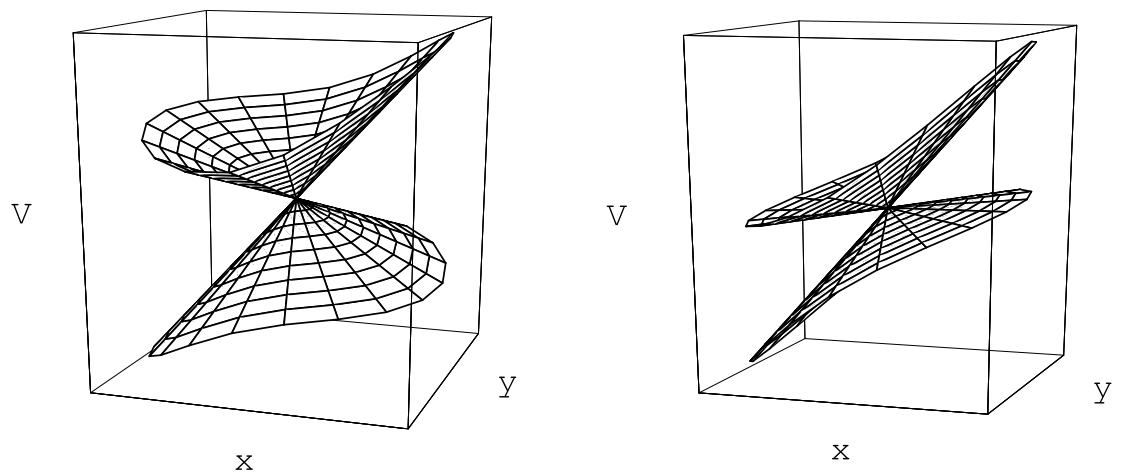

Figure 1. Potential energy surfaces $\mathrm{V}$ as a function of the branching space coordinates $\mathrm{x}$ and $\mathrm{y}$ in the vicinity of different topography CIs, as given by eqs. (1) and (2). The left panel corresponds to a peaked CI with the tilt angle $\alpha_{x}$ equal to $10^{\circ}$. On the right panel $\alpha_{x}=50^{\circ}$, and the CI is sloped. (Figure taken from reference ${ }^{40}$.)

$F$ is a generalized slope on the radial direction, and $\alpha_{x}$ and $\alpha_{y}$ are tilt angles of the planes superimposed to the vertical conical surfaces defined by the radical term of eqs. (1) and (2) $\left(A_{x}\right.$ in eq. (1) equals $F \tan \left(\alpha_{x}\right)$ in in eq. (2), with equivalent expressions along the $y$ axis). If both $\alpha$ angles are smaller than $\pi / 4$, the CI is a local minimum of the excited state potential energy surface within the branching space, and it is a peaked $\mathrm{CI}^{14}$. Conversely, if any of the $\alpha$ angles is greater than $\pi / 4$, the CI is sloped ${ }^{14}$ (see Figure 1 ). The parameters $\left\{e, F, \alpha_{x}, \alpha_{y}\right\}$ are specific to a given CI point, and in moving along the seam, although the degeneracy is not lifted, these parameters can change.

\section{A. Probability for a single trajectory}

In order to derive an expression for the non-adiabatic transition probability, we adopt a quasi-classical perspective in which the nuclear motion is described by a classical trajectory. In addition, we consider the case where the system passes in a straight-line trajectory in the vicinity of a generic CI where the potential energy surfaces' description via eq. (2) applies. Along such a trajectory, the adiabatic energy profile of the two states which are degenerate at the CI corresponds to a cut on the double cone surface by a vertical plane, and thus corresponds to an hyperbola (see Fig. 2 and the Appendix).

The probability of a non-adiabatic transition for a system described by a classical trajectory tracing an hyperbolic adiabatic energy profile in time - or equivalently with an 



Figure 2. The adiabatic energy profile for a straight line trajectory on the branching space for potential energy surfaces given by eq. (1) (or equivalently by eq. (2)) is hyperbolic. The upper panel represents the straight line trajectory in the branching plane in the vicinity of the CI, where the contours denote the potential energy gap between the surfaces. The lower panel represents a vertical cut on the double cone potential highlighting the hyperbolic profile.

hyperbolic profile in space under the condition of constant velocity - is given by the LZ equation

$$
P=\exp \left(-\frac{\pi \Delta V_{\min }^{2}}{2 \hbar \Delta S v}\right)
$$

where $\Delta V_{\min }$ is the smallest adiabatic energy gap along the trajectory, $\Delta S$ is the slope difference of the two asymptotes defining the hyperbolic energy profile, and $v$ is the magnitude of the velocity (assumed constant in the derivation ${ }^{24,25}$ ) at which the CI region is traversed. In the specific case of a straight line trajectory in the vicinity of the double cone given by eq. (2), these parameters take the following form (see the Appendix for the derivation)

$$
\Delta V_{\min }=2 F r \sqrt{\frac{e}{\sin ^{2}(\theta)+e \cos ^{2}(\theta)}},
$$




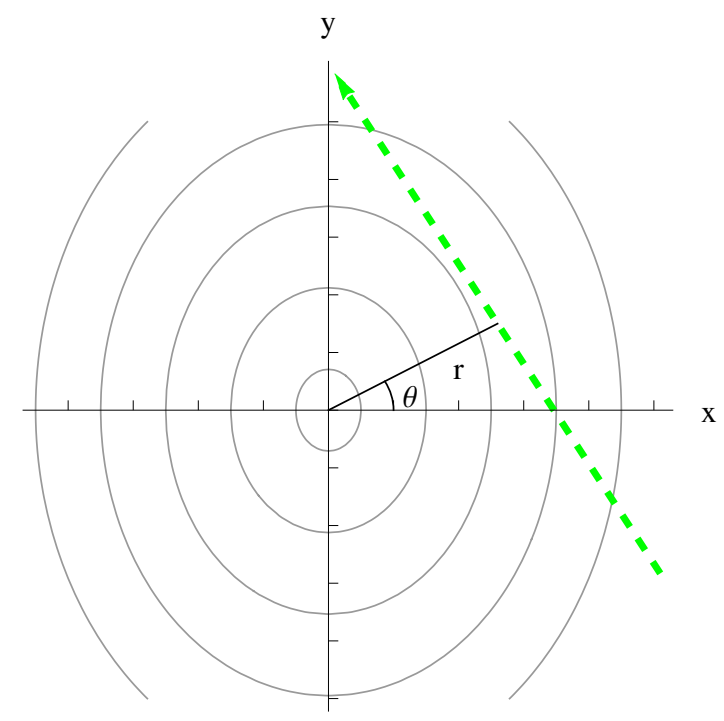

Figure 3. Representation of a straight-line trajectory (green dashed line) in the vicinity of a generic $\mathrm{CI}$ at the center of the axis system. The contour lines correspond to the potential energy difference for the surfaces given by eq. (1) (or eq. (2)) and eccentricity parameter $e=0.5$. The coordinates $r$ and $\theta$ define the trajectory's point of closest approach to the CI (see the text and the Appendix); and although they do not specify the trajectory's orientation (from negative to positive $y$ or vice versa), this is unimportant since both trajectories would predict the same transition probability according to the LZ equation (3) for the same velocity magnitude.

and

$$
\Delta S=2 F \sqrt{\sin ^{2}(\theta)+e \cos ^{2}(\theta)}
$$

where, as illustrated in Fig. 3, $r$ determines the distance of the closest point to the CI along the trajectory, and $\theta$ determines the direction of the trajectory: $\theta=0$ corresponds to a trajectory parallel to the $y$ axis, while the trajectory in the upper panel of Fig. 2 corresponds to $\theta=3 \pi / 2$.

Inserting expressions eqs. (4) and (5) into the LZ eq. (3), we obtain the probability of non-adiabatic transition for an individual trajectory as

$$
P=\exp \left(-\frac{\pi F r^{2}}{\hbar v} \Theta(\theta, e)\right)
$$

with

$$
\Theta(\theta, e)=\frac{e}{\left(\sin ^{2}(\theta)+e \cos ^{2}(\theta)\right)^{3 / 2}} .
$$






Figure 4. Variation of the directional parameter $\Theta$, defined in eq. (7), as a function of the values of the angle $\theta$ and the ellipticity parameter $e$.

These equations depend on the trajectory's velocity $v$, distance of closest approach to the CI apex $r$ and the trajectory orientation $\theta$, as well as the surfaces slope and ellipticity parameters $F$ and $e$. It is a crucial feature that the potential energy gap eq. (4) or the difference in asymptote slope eq. (5) do not depend on the tilt angles $\alpha_{x}$ and $\alpha_{y}$, with the key result that the non-adiabatic transition probability eq. (6) is therefore also independent of these quantities. Equation (6) is equivalent to Nikitin's result ${ }^{29,30}$ originally derived for a vertical CI, but it has been here demonstrated via a geometrical argument to be valid for a general CI. This independence on the tilt angles is a result of considerable significance: it indicates that - within the approximate treatment specified - the non-adiabatic transition probability of a straight-line trajectory in the vicinity of a CI does not depend on whether the CI is sloped or peaked. This (perhaps surprising) result will be explored further in the following sections.

The function $\Theta$, defined in eq. (7) and represented in Fig. 4, determines the dependence of the transition probability eq. (6) on the direction of the trajectory $\theta$. When the parameter $e$ controlling the elliptical deformation of the surfaces equals unity, the potential energy gap has cylindrical symmetry (see the top portion of Fig. 2), $\Theta$ is equal to one and there is no dependence on the trajectory direction. For $e$ values differing from unity, $\Theta$ is a function with the period $\pi$ in the angle $\theta$. 


\section{B. Probability for a distribution of trajectories}

In the preceding section, we derived an expression for the non-adiabatic transition probability for a single trajectory passing in the vicinity of the CI. But in order to represent a photochemical process, a single trajectory is obviously not representative and the behaviour of a given population distribution must be considered. The details of such a population distribution will depend on the characteristics of how the excited state is formed, and how it evolves in time before reaching the CI region. Here we will derive an expression for the transition probability in the case where the population distribution is driven towards the CI keeping a relatively compact form, generically described by a gaussian distribution of the form

$$
\varrho\left(x_{r}, y_{r}\right)=\frac{1}{2 \pi \sigma_{\|} \sigma_{\perp}} e^{-\frac{y_{r}^{2}}{2 \sigma_{\|}^{2}}} e^{-\frac{x_{r}^{2}}{2 \sigma_{\perp}^{2}}}
$$

where $x_{r}$ and $y_{r}$ are new coordinates in a rotated cartesian system such that $y_{r}$ is aligned with the direction of the propagation of the trajectories, $x_{r}$ is orthogonal to it (see Fig. 5), and $\sigma_{\|}$and $\sigma_{\perp}$ are the respective widths of the distribution in each of these directions.

We will consider the case where the trajectory distribution does not change shape as it crosses the CI region. This implies trajectories have a uniform constant velocity $v$, and are rectilinear with a uniform direction of approach $\theta^{41}$, aspects consistent with our previous assumptions for an individual trajectory. In these circumstances, transition probabilities for each trajectory are thus adequately described by eq. (6) and the non-adiabatic transition probability of a distribution of trajectories can be expressed as a simple integration. The width of the distribution along $y_{r}$ implies only that different trajectories cross the CI region in different moments in time, and does not affect the transition probability of individual trajectories given by eq. (6). The population distribution in eq. (8) can thus be integrated along this coordinate to yield a one dimensional population distribution along the coordinate $x_{r}$ independent of the width $\sigma_{\|}$(see Fig. 5). The axis $x_{r}$ defines the distance of closest approach to the CI apex (compare Figs. 3 and 5), and the integral along this coordinate can be decomposed into two integrals in $r$ for $\theta$ and $\theta+\pi$. Since $\Theta$ in eq. (7) has a period of $\pi$ in $\theta$, we have $P(\theta)=P(\theta+\pi)$, and the following simple integral is obtained

$$
\bar{P}=2 \int_{0}^{\infty} P(r, \theta, v) \varrho(r) d r=\frac{2}{\sqrt{2 \pi} \sigma_{\perp}} \int_{0}^{\infty} e^{-\frac{\pi F r^{2}}{\hbar v} \Theta(\theta, e)} e^{-\frac{r^{2}}{2 \sigma_{\perp}}} d r
$$




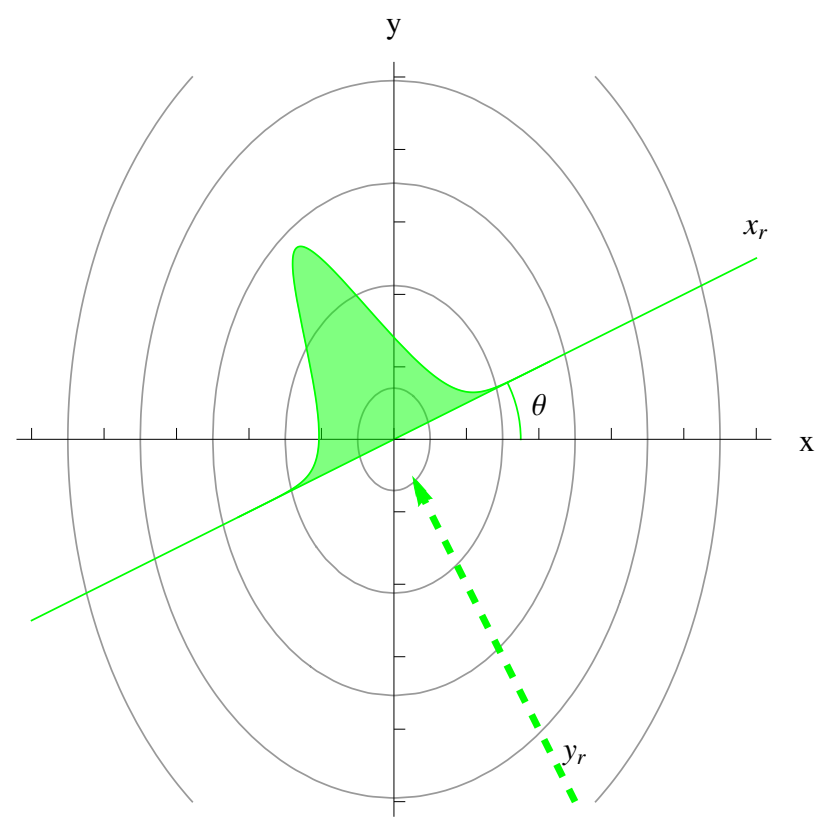

Figure 5. Schematic representation of a gaussian distribution of trajectories travelling through a CI. The contours correspond to the potential energy gap. The angle $\theta$ defines the direction of approach of the distribution, and $y_{r}$ and $x_{r}$ are rotated cartesian coordinates in the direction of approach to the CI and orthogonal to it respectively.

Finally, effecting the gaussian integral gives

$$
\bar{P}=\sqrt{\frac{1}{1+\pi \tilde{\xi} \Theta(\theta, e)}},
$$

with the definition

$$
\tilde{\xi}=\frac{2 \sigma_{\perp}^{2} F}{\hbar v} .
$$

The parameter $\tilde{\xi}$ depends on the ratio of the width squared and the velocity of the population distribution. $\tilde{\xi}$ can be seen as an effective Massey parameter ${ }^{30,42,43}$, an interpretation that follows from the features that $\sigma_{\perp}$ is the average distance of closest approach of the trajectories to the $\mathrm{CI}, 2 \sigma_{\perp} F$ is, from eq. (4), the distribution's average minimum energy gap for a circular CI with the ellipticity $e=1$ ( or $\Theta=1$ ), and that $2 F$ is, from eq. (5), the slope difference in that case.

Equation (10) for the non-adiabatic transition probability is the main result of this contribution. Figure 6 displays this probability's predicted variation as a function of the effective Massey parameter $\tilde{\xi}$ and the directional parameter $\Theta$ for a distribution of trajectories passing through a CI. For any value of $\Theta$, the transition probability will decrease with increasing 


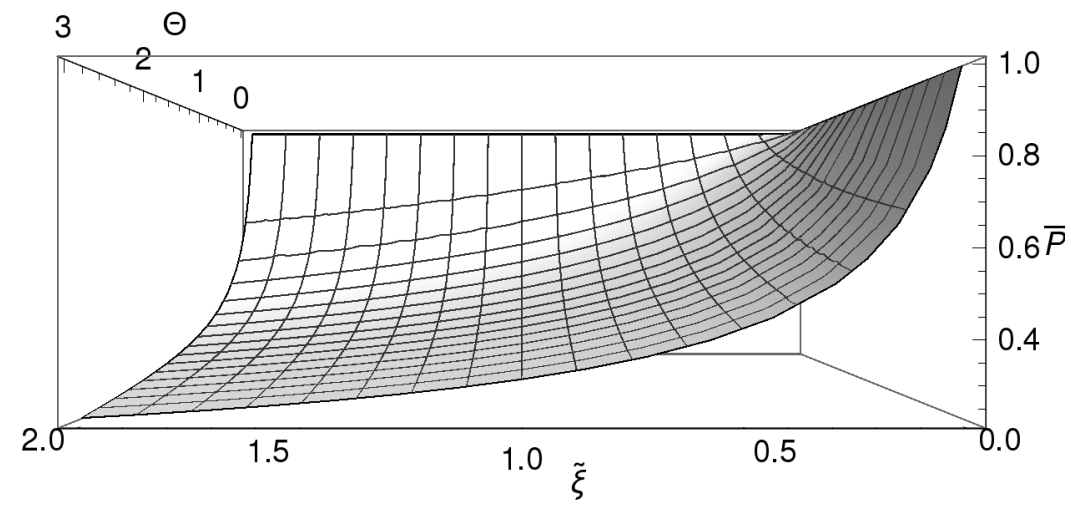

Figure 6. Non-adiabatic transition probability eq. (10) for a distribution of trajectories as a function of the parameters $\tilde{\xi}$ and $\Theta$.

$\tilde{\xi}$, indicating that narrower distributions travelling faster over the CI will result in a higher decay probability. This behaviour is expected, since a narrower distribution will result in trajectories having on average a smaller minimum potential energy gap. An additional feature is that, when the velocity of the distribution tends to zero, the transition probability tends to zero and a fully adiabatic behaviour is predicted; this applies even if there is a degeneracy at the centre of the distribution, since a single degenerate point has a zero measure with respect to the distribution width.

\section{TRANSITION PROBABILITY DEPENDENCE ON THE CI AND POPULATION DISTRIBUTION PROPERTIES}

In this section we will analyze the variation of the non-adiabatic transition probability predicted by eq. (10) as a function of the potential energy surfaces' characteristics at the $\mathrm{CI}$ and the properties of a system described by an ensemble of classical trajectories, and compare these results with surface hopping computer simulations. In these simulations the electronic state of each trajectory is numerically propagated in time, while the nuclear degrees of freedom move classically under the influence of the adiabatic potential surfaces, in this case given by eq. (2), and thus are not restricted to the straight-line assumption under which eq. (10) was derived.

The surface hopping simulations were performed according to the fewest switches algorithm ${ }^{4-46}$ using 10000 independent trajectories. The dimensions of the branching 
space coordinates $x$ and $y$ determine the dimensions of all the parameters in eqs. (2), (10) and (11). In order to study the topography of the potential energy surfaces, the use of mass-weighted coordinates is most convenient, as it allows treating the two branching space coordinates on equal footing from the dynamical point of view without worrying about different masses associated to different degrees of freedom. For illustration purposes, we will

consider that $x$ and $y$ are linear mass-scaled coordinates with units of $\AA . \mathrm{g}^{\frac{1}{2}} \cdot \mathrm{mol}^{-\frac{1}{2}}$, and a double cone slope $\mathrm{F}$ with the value $0.38 \mathrm{eV} . \AA^{-1} \cdot \mathrm{g}^{-\frac{1}{2}} \cdot \mathrm{mol} \frac{1}{2}$, which is consistent with a force acting on a stretching coordinate in an organic molecule with a relatively small distortion from equilibrium. Further details about the initial conditions are given below.

\section{A. Tilt angle independence}

As we have emphasized, a key feature of eq. (10) is that it predicts a non-adiabatic transition probability independent of the tilt angles $\alpha_{x}$ and $\alpha_{y}$ of the potential energy surfaces eq. (2). This is equivalent to stating that, for the same population distribution velocity, a sloped or peaked CI would result in the same non-adiabatic transition probability. This prediction was tested by performing several surface hopping simulations for a wide range $\alpha_{x}$ angle values.

For these simulations, trajectories were started on the excited state surface with a positive velocity along the $x$ axis, $\theta=3 \pi / 2$ (see Fig. 3), with a delta distribution in the positions along $x$, and with a randomly sampled gaussian distribution along $y$. Initial velocities, taken to be the same for each trajectory, were chosen such that, for different $\alpha_{x}$ values, they would result in the same velocity when reaching the CI point ${ }^{47}$. Several different sets of initial velocities and $y$ position distribution widths were examined corresponding to different $\tilde{\xi}$ values shown in Fig. 7. For all these cases, the ellipticity parameter $e$ was set to one, therefore the function $\Theta$ in eq. (10) is also equal to unity (the effect of the parameter $e$ will be studied in the next section).

Figure 7 shows that the transition probability resulting from surface hopping simulations is largely independent of the CI tilt angle, in good agreement with our theoretical prediction. There is a downward trend in the transition probability as the tilt angle increases in the lowest $\tilde{\xi}$ cases. These cases correspond to the lowest kinetic energy and the widest population distribution examined, respectively, blue on the left panel and green on the right panel of 

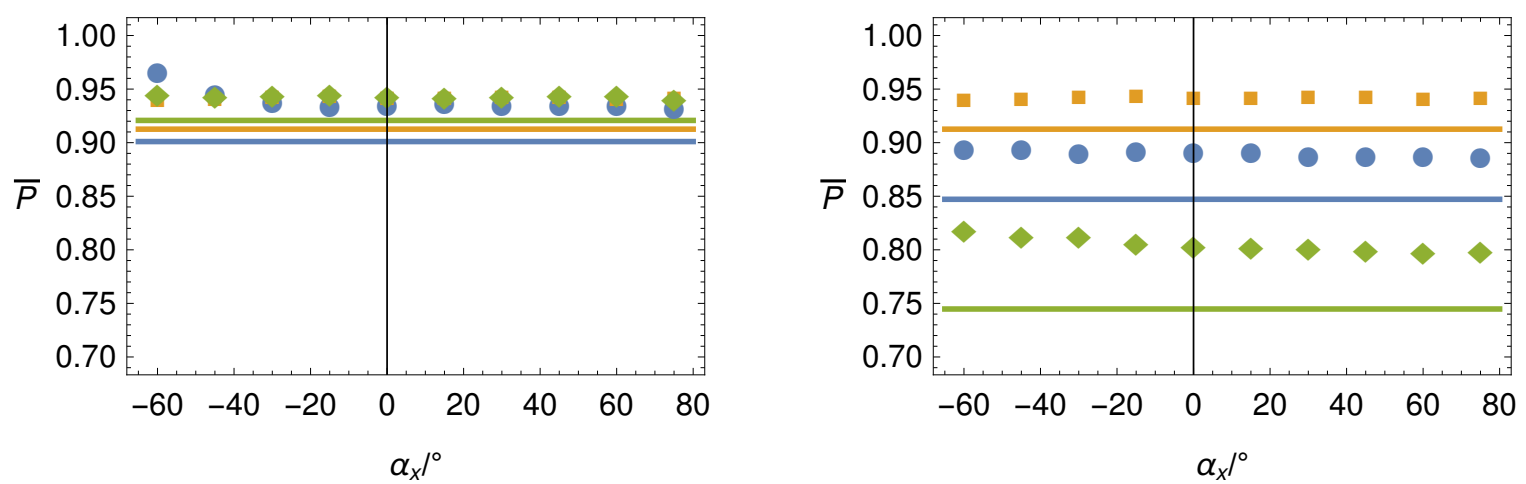

Figure 7. The non-adiabatic transition probability as a function of the tilt angle of the CI with ellipticity parameter $e=1$. For values of tilt angle $\alpha_{x}<-45^{\circ}$ the CI is sloped with trajectories approaching the CI from the steep side of the excited state surface; for the tilt angle range $-45^{\circ}<$ $\alpha_{x}<45^{\circ}$ the $\mathrm{CI}$ is instead peaked; and for the tilt angle values $\alpha_{x}>45^{\circ}$ the $\mathrm{CI}$ is again sloped, with trajectories involving an uphill journey towards the CI. The lines correspond to the eq. (10) prediction, while markers correspond to the fraction of excited state trajectories that transfers to the ground state after one pass in the CI region in surface hopping simulations. On the left hand panel the population distribution width is fixed at $\sigma_{\perp}=0.076 \AA \mathrm{g}^{\frac{1}{2}} \cdot \mathrm{mol}^{-\frac{1}{2}}$, with trajectories distributions set up to reach the CI with different velocities corresponding to $\tilde{\xi}=0.057$ (green), $\tilde{\xi}=0.063$ (orange) and $\tilde{\xi}=0.074$ (blue). On the right panel the total energy of the trajectories is fixed at $2 \mathrm{eV}$ above the CI point, and the with of the population distribution was changed to yield different $\tilde{\xi}$ values: $\tilde{\xi}=0.063$ (orange), $\tilde{\xi}=0.125$ (blue) and $\tilde{\xi}=0.256$ (green).

Fig. 7. The higher values of transition probabilities at lower tilt angles can be explained by the fact that the transition distribution does not conserve a constant width and narrows as it approaches the CI (see Fig. 8). This effect is more prominent for negative tilt angle values, where the trajectories start with a lower kinetic energy and accelerate to reach the CI, and thus are more sensitive to the effect of the excited state potential energy surface that distorts the distribution.

Of greater significance than the slight decrease of transition probability with increase in tilt angle in Fig. 7 is the systematic underestimation of this quantity by eq. (10) compared to surface hopping simulations ${ }^{48}$. In fact, the LZ equation itself (eq. (3)) usually underestimates the transition probability when compared to numerical quantum dynamics simulations ${ }^{49}$ and surface hopping simulations ${ }^{50}$. But here it is seen to be nonetheless useful in reproducing the 

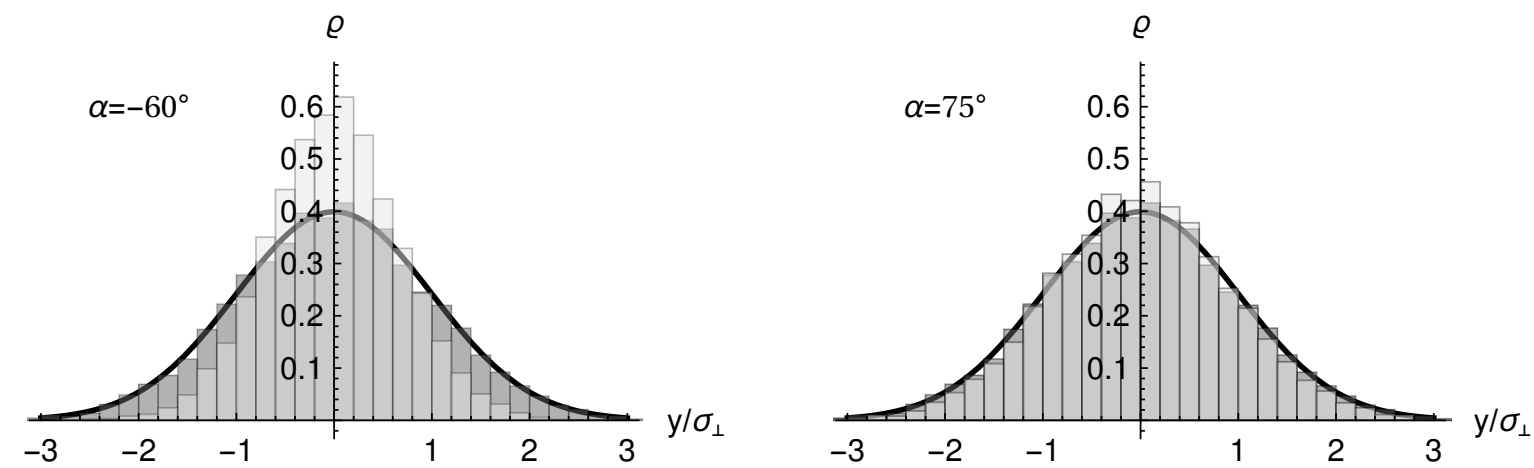

Figure 8. Probability distribution functions of the positions along the $y$ axis for the case $\tilde{\xi}=0.074$ (blue on the left panel of Fig. 7), corresponding to a total trajectory energy of $1.5 \mathrm{eV}$ above the CI point and a population distribution width $\sigma_{\perp}=0.076 \AA \mathrm{g}^{\frac{1}{2}} \cdot \mathrm{mol}^{-\frac{1}{2}}$. In the left and right panels the tilt angle is $\alpha_{x}=-60^{\circ}$ and $\alpha_{x}=-75^{\circ}$ respectively. The solid line corresponds to the gaussian probability distribution in positions, the darker histograms correspond to the sampled initial positions in the surface hopping simulations, while lighter histograms correspond to the excited state distribution in positions just before the CI region is reached.

correct trends, in addition to importantly predicting the equivalence of peaked and sloped CIs in terms of non-adiabatic transition probability.

\section{B. Dependence on CI ellipticity and direction of approach}

In addition to the dependence on the tilt angle just investigated, another important topographical feature of the CI is the elliptical deformation of the surfaces determined by the eccentricity parameter $e$. This parameter controls the potential energy surfaces' deviation from cylindrical symmetry; it therefore determines the transition probability's dependence on the direction of approach to the CI, which enters eq. (10) via the function $\Theta$.

Figure 9 shows the difference in the transition probability of two population distributions that approach the CI from different directions but which are otherwise identical. A clear dependence on the direction of approach correctly predicted by eq. (10) is seen: a higher transition probability is observed for trajectories distributions approaching the CI along the direction where the potential energy gap diminishes faster for the same velocity magnitude ${ }^{51}$.

In contrast to Fig. 7, no systematic shift is observed between eq. (10)'s predictions and 


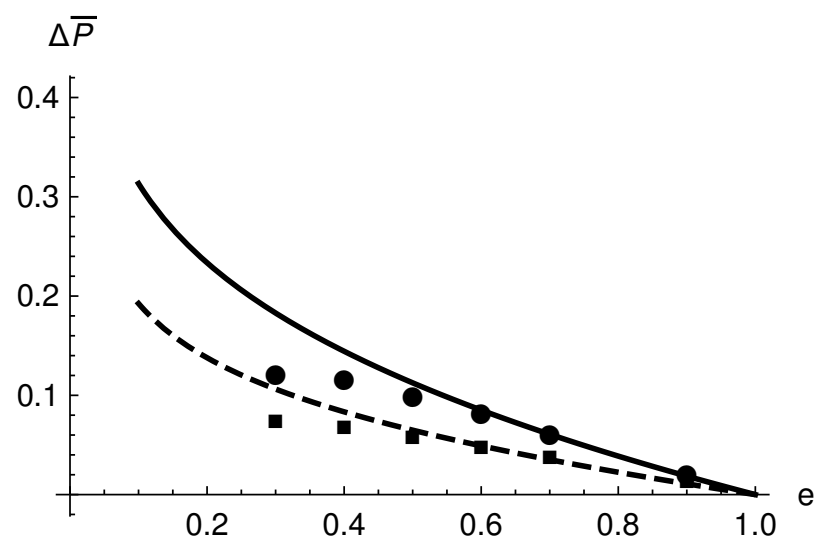

Figure 9. The difference in the transition probability for two identical initial population distributions approaching the CI from 2 different directions $(\Delta \bar{P}=\bar{P}(\theta=3 \pi / 2)-\bar{P}(\theta=0))$ on elliptically distorted potential surfaces $(e \neq 1)$. For the values of $e<1$ shown, $\theta=3 \pi / 2$ corresponds to a direction where the potential energy gap varies more steeply in space, while $\theta=0$ corresponds to an approach with the least steep energy gap variation (see eq. (2) and Fig. 3). The circles and the solid line correspond to $\tilde{\xi}=0.125$, while the squares and the dashed line correspond to $\tilde{\xi}=0.057$.

the surface hopping simulation results, suggesting a likely uniform cancellation of errors when taking the probability difference. For ellipticity $e$ values between one and 0.6, eq. (10) and the simulation results are in good quantitative agreement. On the other hand, eq. (10) overestimates the probability dependence on the direction of approach for smaller $e$ values, arise from the feature that the simulations indicating a higher probability decay than predicted in the case where $\theta=0$. This is due to a distortion of the population distribution analogous to the one described in section III A: for such small $e$ values, the potential energy surfaces have a significant elliptical distortion and become rather steep. This will result in large forces acting on the tails of the population distribution of the surface hopping trajectories with $\theta=0$, which leads to curved - rather than straight - trajectories and narrower population distributions in the approach to the CI. This effect can be observed in Fig. 10, where, for $e=0.3$, the width of an incoming population distribution with $\theta=3 \pi / 2$ remains relatively constant, while a distribution with $\theta=0$ becomes narrower as it approaches the CI. It could be tempting to use eq. (10) with a smaller population distribution width adjusted to describe such situation with a higher non-adiabatic transition probability. However, the narrowing of the population distribution in the vicinity of the CI is the result of curved trajectories in the vicinity of the CI, which violate the assumption of 



Figure 10. Probability distribution functions of the positions along the axis orthogonal to the direction of motion for the cases where $\theta=3 \pi / 2$ and $\theta=0$ on the left and right panels respectively. The solid line corresponds to the gaussian probability distribution in positions, the darker histograms correspond to the sampled initial positions in the surface hopping simulations, while the lighter histograms correspond to the excited state distribution in positions just before the CI region is reached at $x=0$ in the first case and just before $y=0$ in the latter case.

straight-line trajectories which underlies the equation itself.

The ellipticity parameter $e$ indicates differences in potential energy gap slopes in different internal coordinate modes in the CI vicinity. Such differences in mass-weighted coordinates (which we have used throughout our development), are in part due to the differences in reduced mass associated with each mode; these latter differences can have an important effect on the slope of the potential energy surfaces and influences on the relevant CI topography ${ }^{21}$. Equation (10) predicts that, all other factors being equal, the system approaching the CI via a mode with higher reduced mass will have a lower non-adiabatic transition probability. This effect of the mass on the transition probability via the CI topography in mass-scaled coordinates — more precisely, its ellipticity — should in general be considered along with the more direct inertial effects on the dynamics that determine the time-scale of the approach to the $\mathrm{CI}^{21,52}$.

\section{CONCLUDING REMARKS}

Conical intersections (CIs) play a key role in non-adiabatic transitions in a photochemical context. Because of the dimensionality of the branching space a CI is a zero measure manifold 
in the space of system coordinates, and virtually all trajectories attempting a transition between electronic states will pass in close vicinity to, but not exactly through, the CI. In this contribution we have derived a closed form expression eq. (10) - based on the single trajectory Landau-Zener (LZ) equation - for the probability of non-adiabatic transitions for a gaussian distribution of straight-line trajectories in the branching plane passing through a CI. The transition probability depends both on the properties of the trajectory distribution (decreasing with its width $\sigma_{\perp}$, increasing with its velocity $v$, and depending on direction of approach $\theta$ to the CI), as well as the topographical properties of the CI (the overall slope of the potential energy surfaces measured by the parameter $F$ in equation (2), and the eccentricity parameter $e$ ). The probability depends most sensitively on the distribution width $\sigma_{\perp}$ (see eq. (11)), which represents the average minimum distance to the CI apex.

The most noteworthy feature of eq. (10) is that the transition probability shows no dependence on the CI's tilt angles $\alpha_{x}$ and $\alpha_{y}$ (see section III A), which predicts that peaked and sloped CIs have the same transition probability if all other variables are kept the same. This result is in apparent contradiction with the commonly held view that peaked CIs are more efficient at promoting non-adiabatic decay than sloped CIs. But there is no contradiction concerning which type of CI is more efficient, rather it is in the reason for this difference in efficiency that our result brings a specific new insight: it is not because the probability of decay is itself higher in peaked CIs due to a topographical dependence, but rather it is for exclusively dynamical (and intuitively clear) reasons by which peaked CIs, corresponding to local minima of the excited state potential energy surface, act as efficient "funnels" to bring the system towards the CI apex, while for sloped CIs the system is steered away from it (see Fig. 1).

A second important result that follows from eq. (10) reveals an effect of the CI topographic characteristics on the transition probability. It is that in the presence of an elliptical deformation of the surfaces - an eccentricity parameter $e$ different from unity - the transition probability depends on the direction of the approach to the CI, with a higher transition probability being obtained along the direction along which the surfaces energy gap have a steeper variation (see section III B). Since the mass scaling of the coordinates affects the value of the $e$ parameter, if all other variables are equal, an approach to the CI via a coordinate with a smaller associated mass gives rise to a higher transition probability.

The conclusions just stated regarding the transition probability dependence on the CI 


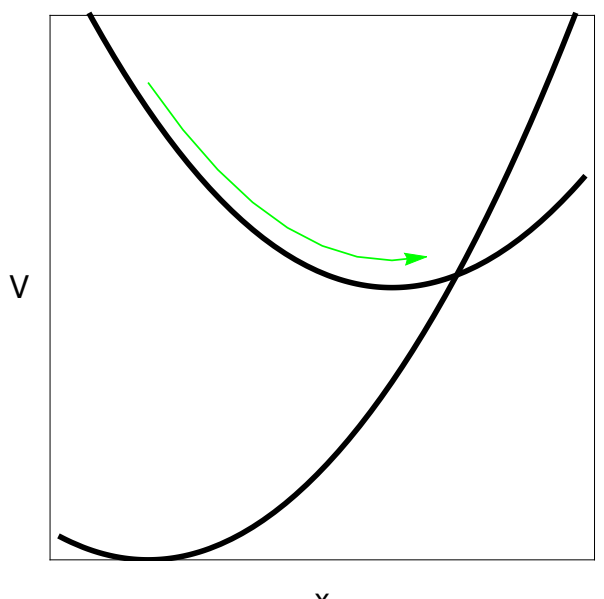

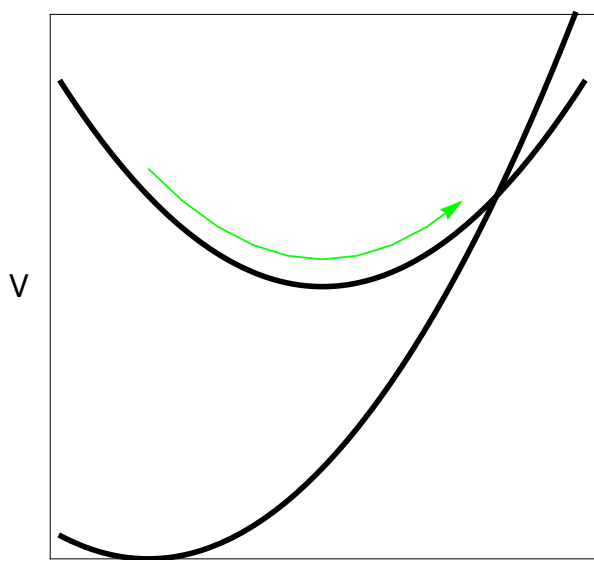

Figure 11. Profile of the potential energy of two sloped CIs. On the left the Franck-Condon region is much higher in energy than the CI and the system reaches it with a higher kinetic energy than on the right-hand side.

topography, both regarding the tilt angles and elliptic deformation, assume the same velocity of approach on the branching space coordinates. Although the local topography may have some influence on the velocity of approach to the CI, this velocity will depend for the most part on the dynamics of the system prior to reaching the CI region, in particular when the Franck-Condon region is high in energy compared to the relevant point along the CI seam and there is a significant amount of kinetic energy on the branching space coordinates (see Fig. 11). In these circumstances, peaked and sloped CIs should remain indistinguishable from the point of view of the non-adiabatic transition probability. At lower kinetic energies however, the local CI topography will have a greater effect on the velocity of the system, with distinct forces acting on the system whether the CI is peaked or sloped and depending on the direction of approach. Real photochemical problems obviously present a range of possibilities. Often the CI is located at lower energy than the Franck-Condon-accessed site on the excited state surface, and - provided that there is no barrier and that not too much energy is transferred out of branching space coordinates - the relevant kinetic energy will not be small. The opposite limit would be where activated barrier crossing is required for the CI to be reached, so that minimal excess kinetic energy could be involved in the barrier passage. Since the low kinetic energy regime is potentially the most problematic, we now devote some further discussion of it. 


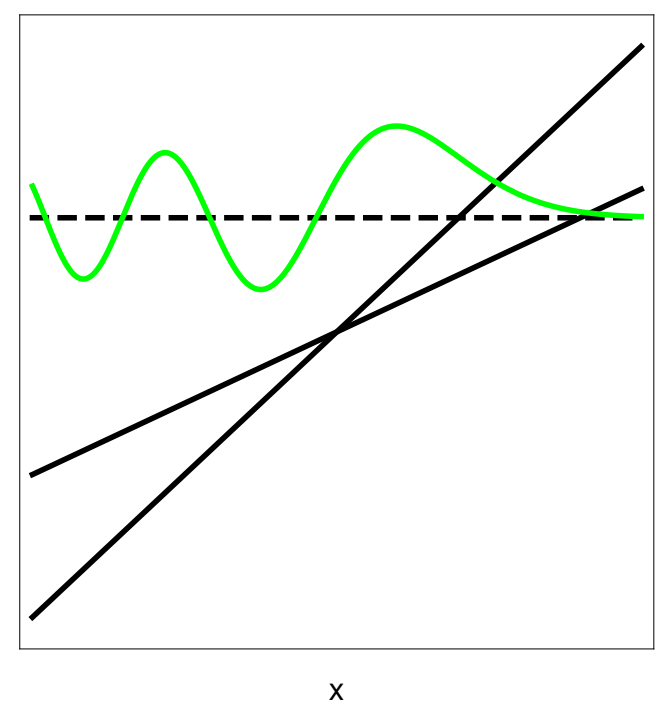

Figure 12. Schematic representation of wavefunction tunnelling for a sloped CI. The wavefunction is represented in green and the profile of the potential energy in black. Note that due to quantum mechanical tunnelling, not taken into account by eq. (10), the probability of non-adiabatic transition is non-zero even if the energy of the system is below the energy of the CI.

In the low kinetic energy limit, when the velocity magnitude can no longer be considered constant while crossing the CI region, the assumptions under which the LZ equation and eq. (10) are derived break down. In such circumstances, a more sophisticated semi-classical treatment of the nuclear degrees of freedom is necessary ${ }^{29,30,42}$, yielding for the peaked and sloped cases two distinct equations for the transition probability. Such difference is associated with the quantum tunnelling effect between the two adiabatic states which can become important for sloped CIs (see Fig. 12), thus predicting that sloped CI could indeed in some situations induce a higher probability of non-adiabatic decay. Zhu and Nakamura ${ }^{49,53}$ have derived distinct closed form expressions, applicable for the peaked and sloped case, which take into account such effect and could be used in a refined treatment along the same lines discussed in the present article. Still, the Zhu-Nakamura equations have the LZ equation as a limiting case for high velocities, agreeing with the results presented here in this limit ${ }^{54}$.

In order to assess how the LZ equation predictions are affected at low kinetic energies, we compare in Fig. 13 the non-adiabatic transition probability calculated by the LZ and ZhuNakamura equations for a sloped CI for trajectories passing at different distances from the CI apex with different kinetic energies. It is seen that the LZ equation produces satisfactory 


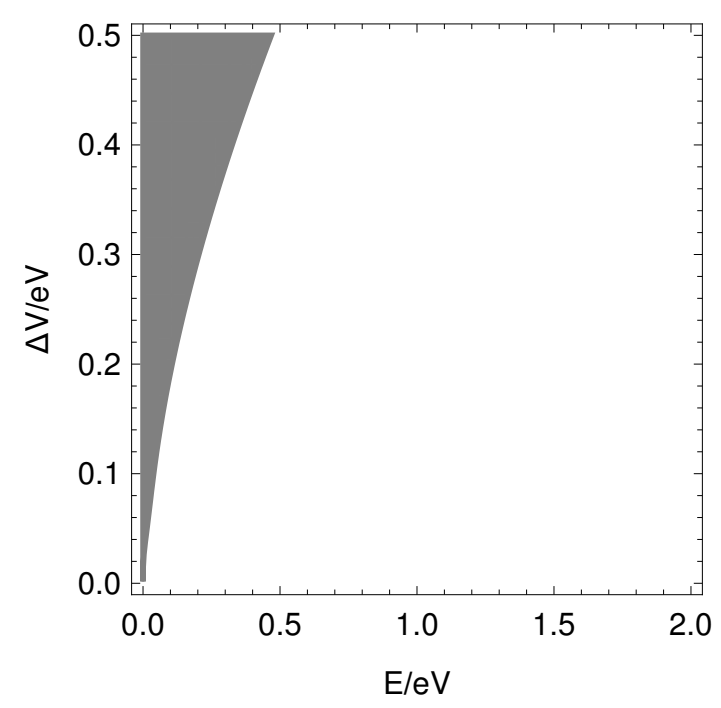

Figure 13. The gray area in the figure represents combinations of trajectory kinetic energy and the energy gap $\Delta V$ at the point of closest approach to the CI for which the LZ eq. (6) predicts a transition probability with an error greater than $10 \%$ with respect to the appropriate Zhu-Nakamura equation $^{49,53}$. The case considered corresponds to tilt angles $\alpha_{x}=55^{\circ}, \alpha_{y}=0^{\circ}$, ellipticity $e=$ 1, direction of approach $\theta=3 \pi / 2$ and a generalized sloped $F$ value equal to that used in the simulations shown in Sec. III.

predictions for kinetic energies above tenths of $\mathrm{eV}$, especially in the center of the population distribution, closer to the CI, which is the region that contributes the most for the total transition probability.

The electron dynamics of the system is also affected by the decoherence effects ${ }^{40,55,56}$ by which the off-diagonal elements of the electronic density matrix tend to zero with time due to dephasing and divergent wavepacket motions on different electronic states. This is a quantum effect not taken into account by either eq. (10) or the surface hopping algorithm used; its neglect can lead to artifacts when several passages through regions of strong adiabatic coupling are considered ${ }^{57,58}$. This is less of a concern in the present case of a single passage over the CI, with population transfer occuring coherently in a short time scale ${ }^{56,59,60}$. However, for small values the generalized slope parameter $F$ and very flat surfaces, electronic population transfer can occur in extended regions of space and time, decoherence may become important, and the use of the LZ model may no longer be appropriate.

An additional quantum effect not taken into account by eq. (10) is the geometric phase characteristic of the CI topology ${ }^{32,61-63}$. A previous study ${ }^{64}$ has however shown that methods 
that describe the nuclear motion classically, like the one described here, are able to reproduce well the transition probabilities calculated by quantum mechanical methods that include geometric phase.

A final observation concerns the full dimensionality of the system. We have focussed in this article on the properties of the dynamics of the system in the two dimensions of the branching space and on a specific CI point where all topographical parameters in eqs. (2) and (10) have well defined values. One must however consider a population distribution in the remaining degrees of freedom that constitute the intersection space, and that the system traverses a section of the CI seam instead of a CI point. Due to the curvature of the CI seam $^{65}$, a distribution of parameters should be considered, and eq. (10) should be used with suitable averaged values.

Despite the restrictions we have indicated, eq. (10) should nevertheless prove useful in describing general trends in the major factors affecting the non-adiabatic transition probabilities at CIs predicting the correct behaviour when the nuclear motion is described classically, as shown in comparison of the predictions we have made with the simulations results in section III.

\section{ACKNOWLEDGMENTS}

The authors would like to thank Morgane Vacher for reading and commenting on an early version of the manuscript. This work was supported in part by NSF grant CHE-1112564 (JTH)

\section{Appendix: Potential energy profile for a straight-line trajectory on a general double cone potential}

In this appendix we address the classical geometric problem of the intersection of a vertical plane with a conical surface, showing that the intersection's shape is always an hyperbola. This result's relevance is that any system with a rectilinear trajectory in the vicinity of a conical intersection (CI) will have an hyperbolic potential energy profile, and thus is subject to a probability of non-adiabatic decay which can be expressed by the Landau-Zener (LZ) eq. (3) of the text. This result was first pointed out by Teller ${ }^{2}$ and later adopted by Nikitin ${ }^{29,30}$, 
but only demonstrated for the vertical cone case. Our demonstration is valid for any general double cone. An expression for the asymptote's slope difference (text eq. (5)) and minimum energy along a straight-line trajectory (text eq. (4)), which are both key parameters of the LZ equation, are derived as a function of the geometric properties of the double cone.

A general conical surface with its apex at the reference frame's origin can, in polar cylindrical coordinates $(\rho, \phi, V)$, be given by text eq. (2). A plane can be defined by a vector orthogonal to it, and by definition the vector formed by the point on the place closest to the CI apex/frame origin and this same apex is orthogonal to the plane. We take this point to be $(r, \theta, 0)$ in polar cylindrical coordinates, and note that the values $r$ and $\theta$ define any arbitrary vertical plane (see Fig. 14). It is useful to define a coordinate $q$ along the intersection of the vertical and horizontal $V=0$ planes, measured from the point $(r, \theta, 0)$; such a rectilinear coordinate $q$ is defined along the trajectory path. It follows from Fig. 14 that any point on the vertical plane is defined by the following equations

$$
\left\{\begin{array} { l } 
{ r = \rho \operatorname { c o s } ( \phi - \theta ) } \\
{ \frac { q } { r } = \operatorname { t a n } ( \phi - \theta ) }
\end{array} \Leftrightarrow \left\{\begin{array}{l}
\rho=\frac{r}{\cos (\phi-\theta)} \\
\phi=\arctan \left(\frac{q}{r}\right)+\theta
\end{array},\right.\right.
$$

with $q$ varying from minus infinity to plus infinity and $(\phi-\theta) \in]-\frac{\pi}{2}, \frac{\pi}{2}[$. This angular restriction amounts to considering only the half of the double cone surfaces that intersect the vertical plane and involves no loss of generality.

Upon substitution of the defining plane equations (A.1) into the equation of the conical surfaces (2), an equation defining the cone-plane intersection is obtained as a function of the coordinate $q$ along the plane, the plane parameters $r$ and $\theta$, as well as the cone surface parameters $F, e, \alpha_{x}$ and $\alpha_{y}$

$$
\begin{aligned}
V & =F \frac{r}{\cos \left(\arctan \left(\frac{q}{r}\right)\right)}\left( \pm \sqrt{\cos ^{2}\left(\arctan \left(\frac{q}{r}\right)+\theta\right)+e \sin ^{2}\left(\arctan \left(\frac{q}{r}\right)+\theta\right)}+\right. \\
& \left.\tan \left(\alpha_{x}\right) \cos \left(\arctan \left(\frac{q}{r}\right)+\theta\right)+\tan \left(\alpha_{y}\right) \sin \left(\arctan \left(\frac{q}{r}\right)+\theta\right)\right)= \\
& =F r\left( \pm \sqrt{\left(\cos ^{2}(\theta)+e \sin ^{2}(\theta)\right)+\frac{q}{r} \sin (2 \theta)(-1+e)+\left(\frac{q}{r}\right)^{2}\left(\sin ^{2}(\theta)+e \cos ^{2}(\theta)\right)}+\right. \\
& \left.\tan \left(\alpha_{x}\right) \cos (\theta)+\tan \left(\alpha_{y}\right) \sin (\theta)+\frac{q}{r}\left(-\tan \left(\alpha_{x}\right) \sin (\theta)+\tan \left(\alpha_{y}\right) \cos (\theta)\right)\right)
\end{aligned}
$$




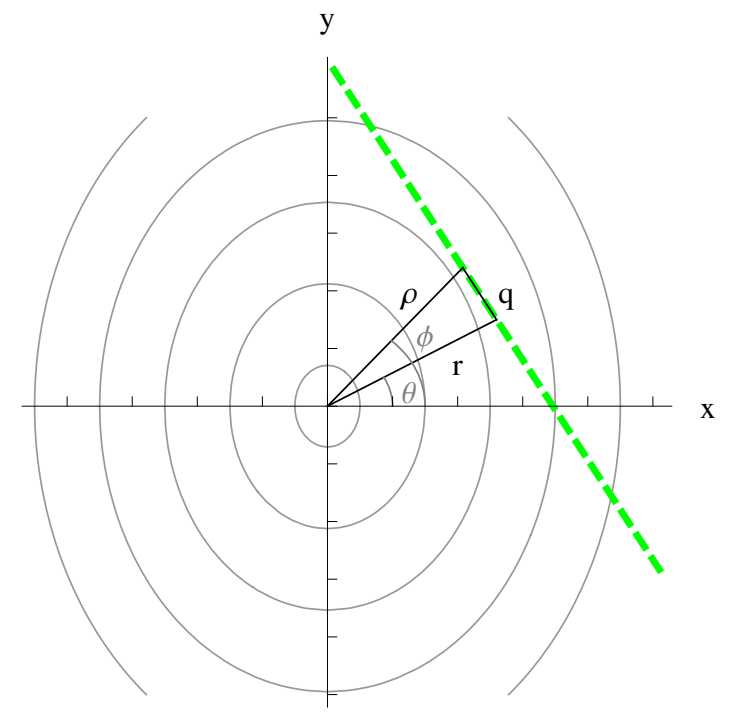

Figure 14. Schematic representation of a conical surface by a vertical plane. The contours represent the potential energy difference for conical surfaces defined by text eq- (2) with the ellipticity parameter $e \neq 1$; the dashed green line is the intersection with the plane oriented along an arbitrary rectilinear trajectory. The coordinates $(\rho, \phi, V)$ define a general point in space, and here we are interested in the points at which the plane and the double cone surface intersect. The point with polar cylindrical coordinates $(r, \theta, 0)$ corresponds to the point of closest approach of the plane with the double cone apex, and together with the axis origin defines a vector orthogonal to the plane, thus defining that plane. $q$ is a rectilinear coordinate along the plane/trajectory, having the value zero at the point of closest approach. Note that for the particular choice of $(\rho, \phi)$, from the contours it is possible to see that the energy gap between conical surfaces at this point is smaller than at the point of closest approach $(r, \theta)$. 
Rearrangement and squaring both sides of the equation yields

$$
\begin{aligned}
& V^{2} \frac{1}{F^{2} r^{2}}+ \\
& V q \frac{2}{F r^{2}}\left(\tan \left(\alpha_{x}\right) \sin (\theta)-\tan \left(\alpha_{y}\right) \cos (\theta)\right)+ \\
& q^{2} \frac{1}{r^{2}}\left(\left(\tan \left(\alpha_{x}\right) \sin (\theta)-\tan \left(\alpha_{y}\right) \cos (\theta)\right)^{2}-\left(\sin ^{2}(\theta)+e \cos ^{2}(\theta)\right)\right)- \\
& V \frac{2}{F r}\left(\tan \left(\alpha_{x}\right) \cos (\theta)+\tan \left(\alpha_{y}\right) \sin (\theta)\right)+ \\
& q \frac{1}{r}\left(\sin (2 \theta)(1-e)-2\left(\tan \left(\alpha_{x}\right) \sin (\theta)-\tan \left(\alpha_{y}\right) \cos (\theta)\right)\left(\tan \left(\alpha_{x}\right) \cos (\theta)+\tan \left(\alpha_{y}\right) \sin (\theta)\right)\right)+ \\
& \left(\tan \left(\alpha_{x}\right) \cos (\theta)+\tan \left(\alpha_{y}\right) \sin (\theta)\right)^{2}-\left(\cos ^{2}(\theta)+e \sin ^{2}(\theta)\right)=0 .
\end{aligned}
$$

This quadratic equation in $V$ and $q$ is of the form $\mathcal{A} V^{2}+\mathcal{B} V q+\mathcal{C} q^{2}+\mathcal{D} V+\mathcal{E} q+\mathcal{F}=0$, which defines a conic section ${ }^{66}$, and the type of conic (ellipse, parabola or hyperbola) is determined $^{66}$ by the equation's discriminant $4 \mathcal{A C}-\mathcal{B}^{2}$, which in the present case takes the form

$$
-\frac{4}{F^{2} r^{4}}\left(\sin ^{2}(\theta)+e \cos ^{2}(\theta)\right)<0
$$

Since the cone elliptical deformation parameter $e$ is an always positive number, the discriminant is always negative, and the double cone's intersection with a vertical plane is always an hyperbola for all parameter values. A consequence of this result is that the non-adiabatic transition probability for a system describing a straight-line trajectory in the vicinity of an arbitrary CI is given by the LZ equation ${ }^{40}$ (within limits of applicability of that equation).

From the equations above, there are two parameters needed to use the LZ text eq. (3), the minimum energy gap along the trajectory $\Delta V_{\text {min }}$, and the difference in the slopes $\Delta S$ of the asymptotes that define the hyperbolic potential profile along the trajectory. $\Delta V_{\min }$ can be determined by differentiating with respect to $q$ the energy gap between the two surfaces obtained from eq. (A.2), and equating it to zero:

$$
\frac{d \Delta V}{d q}=0
$$

with the potential energy gap given by

$$
\Delta V=2 F r \sqrt{\left(\cos ^{2}(\theta)+e \sin ^{2}(\theta)\right)+\frac{q}{r} \sin (2 \theta)(-1+e)+\left(\frac{q}{r}\right)^{2}\left(\sin ^{2}(\theta)+e \cos ^{2}(\theta)\right)} .
$$


Alternatively, the position of the minimum can be obtained in simply geometrical terms from the centre of the hyperbola which is determined by solving the system of equations ${ }^{67}$ :

$$
\left\{\begin{array}{l}
\mathcal{A} V+\frac{\mathcal{B}}{2} q+\frac{\mathcal{D}}{2}=0 \\
\frac{\mathcal{B}}{2} V+\mathcal{C} q+\frac{\mathcal{E}}{2}=0
\end{array},\right.
$$

with the terms defined by eq. (A.3) above. Either of these procedures gives for the position of the minimum

$$
q_{\text {min }}=r \frac{(1-e) \sin (\theta) \cos (\theta)}{\sin ^{2}(\theta)+e \cos ^{2}(\theta)},
$$

and the minimum energy gap

$$
\Delta V_{\text {min }}=2 F r \sqrt{\frac{e}{\sin ^{2}(\theta)+e \cos ^{2}(\theta)}},
$$

which is eq. (4) of the main text. In the more general case, for an elliptic distorted CI where $e \neq 1$, the position of the minimum energy gap is not the same as the point of closest approach to the CI $q=0$ (see Figure 14).

The slopes of the asymptotes of the hyperbola can be obtained from the equation $\mathcal{A} V^{2}+$ $\mathcal{B} V q+\mathcal{C} q^{2}=0$ which is the equation of two lines parallel to asymptotes passing through the point $(q=0, V=0)^{66}$; each of these lines can be written in the form $V=S q$ where $S$ is the asymptote slope. Taking the definition of these terms form eq. (A.3), and determining the slope difference one obtains

$$
\Delta S=2 F \sqrt{\sin ^{2}(\theta)+e \cos ^{2}(\theta)},
$$

which is eq. (5) of the main text.

\section{REFERENCES}

${ }^{1}$ J. von Neuman and E. Wigner, Phys. Z. 30, 467 (1929), English translation in ${ }^{68}$.

${ }^{2}$ E. Teller, J. Phys. Chem. 41, 109 (1937).

${ }^{3} \mathrm{~J}$. Michl and V. Bonačić-Koutecký, Electronic aspects of organic photochemistry (Wiley, 1990).

${ }^{4}$ W. Domcke, D. R. Yarkony, and H. Köppel, eds., Conical intersections: electronic structure, dynamics and spectroscopy (World Scientific Publishing, 2004). 
${ }^{5}$ W. Domcke, D. R. Yarkony, and H. Köppel, eds., Conical intersections:theory, computation and experiment (World Scientific Publishing, 2011).

${ }^{6}$ D. R. Yarkony, Chem. Rev. 112, 481 (2012).

${ }^{7}$ B. G. Levine and T. J. Martínez, Annu. Rev. Phys. Chem. 58, 613 (2007).

${ }^{8}$ F. Bernardi, M. Olivucci, and M. A. Robb, Chem. Soc. Rev. 25, 321 (1996).

${ }^{9}$ A. W. Jasper and D. G. Truhlar, J. Chem. Phys. 122, 044101 (2005).

${ }^{10}$ D. G. Truhlar, in Non-Adiabatic Effects in Chemical Dynamics, Faraday Discussions, Vol. 127 (2004) p. 242.

${ }^{11}$ A. Stolow, Faraday Discuss. 163, 9 (2014).

${ }^{12}$ J. C. Polanyi, Acc. Chem. Res. 5, 161 (1972).

${ }^{13}$ See discussion in Non-Adiabatic Effects in Chemical Dynamics, Faraday Discussion, Vol. 127 (2004) p. 227; and in Non-Adiabatic Effects in Chemical Dynamics, Faraday Discussions, Vol. 127 (2004) p. 445.

${ }^{14}$ G. J. Atchity, S. S. Xantheas, and K. Ruedenberg, J. Chem. Phys. 95, 1862 (1991).

${ }^{15}$ B. C. Hoffman and D. R. Yarkony, J. Chem. Phys. 113, 10091 (2000).

${ }^{16}$ M. Ben-Nun, F. Molnar, K. Schulten, and T. J. Martínez, Proc. Natl. Acad. Sci. USA 99, 1769 (2002).

${ }^{17}$ A. Toniolo, G. Grannucci, and T. J. Martínez, J. Phys. Chem. A 107, 3822 (2003).

${ }^{18}$ B. Lasorne, M. J. Bearpark, M. A. Robb, and G. A. Worth, J. Phys. Chem. A 112, 13017 (2008).

${ }^{19}$ D. Asturiol, B. Lasorne, G. A. Worth, M. A. Robb, and L. Blancafort, Phys. Chem. Chem. Phys. 12, 4949 (2010).

${ }^{20}$ A. M. Virshup, J. Chen, and T. J. Martínez, J. Chem. Phys. 137, 22A519 (2012).

${ }^{21}$ J. P. Malhado and J. T. Hynes, J. Chem. Phys. 137, 22 A543 (2012).

${ }^{22}$ D. R. Yarkony, J. Chem Phys. 114, 2601 (2001).

${ }^{23}$ P. Krause, S. Matsika, M. Kotur, and T. Weinacht, J. Chem. Phys. 137, 22A537 (2012).

${ }^{24}$ L. D. Landau, Phys. Z. Sowjetunion 2, 46 (1932), English translation in ${ }^{69,70}$.

${ }^{25}$ C. Zener, Proc. Roy. Soc. A 137, 696 (1932).

${ }^{26}$ E. C. G. Stückelberg, Helv. Phys. Acta 5, 369 (1932).

${ }^{27}$ E. Majorana, Nuovo Cimento 9, 43 (1932).

${ }^{28}$ For a commentary on the of the different derivations of the Landau-Zener equation, all published in the same year, see reference ${ }^{71}$. 
${ }^{29}$ E. E. Nikitin, "Chemische elementarprozesse," (Springer-Verlag, 1968) Chap. Theory of Non-Adiabatic Transitions. Recent Developement of the Landau-Zener (Linear) Model, pp. $43-77$.

${ }^{30}$ E. E. Nikitin, Theory of Elementary Atomic and Molecular Processes in Gases (Oxford University Press, 1974).

${ }^{31}$ C. Zhu, E. E. Nikitin, and H. Nakamura, J. Chem. Phys. 104, 7059 (1996).

${ }^{32}$ E. E. Nikitin, J. Chem. Phys 107, 6748 (1997).

${ }^{33}$ A. Alijah and E. E. Nikitin, Mol. Phys. 96, 1399 (1999).

${ }^{34}$ M. Desouter-Lecomte, C. Sannen, and J. C. Lorquet, J. Chem. Phys. 79, 894 (1983).

${ }^{35}$ M. Desouter-Lecomte, D. Dehareng, B. Leyh-Nihant, M. Praet, and A. J. Lorquet, J. Phys. Chem. 89, 214 (1985), see also reference ${ }^{72}$.

${ }^{36}$ J. C. Lorquet and B. Leyh-Nihant, J. Phys. Chem. 92, 4778 (1988).

${ }^{37}$ E. Teller, Israel J. Chem. 7, 227 (1969).

${ }^{38}$ C. A. Mead, J. Chem. Phys. 70, 2276 (1979).

${ }^{39}$ S. Matsika and P. Krause, Annu. Rev. Phys. Chem. 62, 621 (2011).

${ }^{40}$ J. P. Malhado, M. J. Bearpark, and J. T. Hynes, Front. Chem. 2, 97 (2014).

${ }^{41} \mathrm{~A}$ population distribution with a varying total width in $x_{r}$ would involve a distribution in the approach direction $\theta$, which could still be treated using eq. (6), or curved trajectories for which eq. (6) becomes unsuitable. For a brief discussion on the effect of curvature on the LZ transition probability in the context of CIs see reference ${ }^{50}$.

${ }^{42}$ M. S. Child, Molecular Collision Theory (Academic Press, 1974).

${ }^{43}$ M. Desouter-Lecomte and J. C. Lorquet, J. Chem. Phys. 71, 4391 (1979).

${ }^{44}$ J. C. Tully, J. Chem. Phys. 93, 1061 (1990).

${ }^{45}$ D. F. Coker, "Computer simulation in chemical physics," (Kluwer Academic Publishers, 1993) Chap. Computer Simulation Methods for Nonadiabatic Dynamics in Condensed Systems, pp. 315-377.

${ }^{46}$ K. Drukker, J. Comp. Phys. 153, 225 (1999).

${ }^{47}$ In surface hopping simulations, the dynamics is determined by the adiabatic potential energy surface of the state in which the trajectory is in each moment in time. Therefore, different trajectories will not reach the CI vicinity with the same velocity, and in general they will not be straight-line trajectories. However, trajectories were started relatively close to the CI point ( $0.76 \AA \cdot g^{\frac{1}{2}} \cdot \mathrm{mol}^{-\frac{1}{2}}$ away), albeit not close enough to induce immediate 
transitions, and with a relatively high velocity magnitude (total energy at least $1.5 \mathrm{eV}$ above the CI energy), such as to reduce deviations in both direction and magnitude of the velocity of each trajectory.

${ }^{48}$ We note that by replacing the factor 2 in the numerator of eq. (11) by $\sqrt{2}$ a much better quantitative agreement is obtained between the predictions of eq. (10) and simulation results. We have however found no legitimate argument for this replacement.

${ }^{49}$ H. Nakamura and C. Zhu, Comments At. Mol. Phys. 32, 249 (1996).

${ }^{50}$ J. P. Malhado and J. T. Hynes, Chem. Phys. 347, 39 (2008).

${ }^{51}$ Teller noted this trend ${ }^{2}$ for vertical CIs based on the LZ transition probability for a single trajectory.

${ }^{52}$ O. Schalk, A. E. Boguslavskiy, and A. Stolow, J. Phys. Chem. A 114, 4058 (2010).

${ }^{53}$ C. Zhu and H. Nakamura, J. Chem. Phys. 101, 4855 (1994).

${ }^{54}$ For another high accuracy explicit formula for the tilted case, see reference ${ }^{73}$.

${ }^{55}$ G. A. Fiete and E. J. Heller, Phys. Rev. A 68, 022112 (2003).

${ }^{56}$ C. Zhu, S. Nangia, A. W. Jasper, and D. G. Truhlar, J. Chem. Phys. 121, 7658 (2004).

${ }^{57}$ M. Thachuk, M. Y. Ivanov, and D. M. Wardlaw, J. Chem. Phys. 109, 5747 (1998).

${ }^{58}$ J. E. Subotnik, A. Jain, B. Landry, A. Petit, and W. O. O. Bellonzi, Annu. Rev. Phys. Chem. 67, 387 (2016).

${ }^{59}$ J.-Y. Fang and S. Hammes-Schiffer, J. Phys. Chem. A 103, 9399 (1999).

${ }^{60}$ G. Granucci and M. Persico, J. Chem. Phys. 126, 134114 (2007).

${ }^{61}$ G. Herzberg and H. C. Longuet-Higgins, Discuss. Faraday Soc. 35, 77 (1963).

${ }^{62}$ M. V. Berry, Proc. R. Soc. Lond. A 392, 45 (1984).

${ }^{63}$ I. G. Ryabinkin, L. Joubert-Doriol, and A. F. Izmaylov, J. Chem. Phys. 140, 214116 (2014).

${ }^{64}$ R. Gherib, I. G. Ryabinkin, and A. F. Izmaylov, J. Chem. Theory Comput. 11, 1375 (2015).

${ }^{65}$ F. Sicilia, L. Blancafort, M. J. Bearpark, and M. A. Robb, J. Phys. Chem. A 111, 2182 (2007).

${ }^{66}$ B. Spain, Analytical conics (Pergamon Press, 1957).

${ }^{67}$ J. W. Rutter, Geometry of Curves (Chapman \& Hall/CRC, 2000).

${ }^{68}$ H. Hettema, ed., Quantum Chemistry: Classic Scientific Papers (World Scientific, 2000).

${ }^{69}$ D. ter Haar, ed., Collected papers of L. D. Landau (Pergamon Press, 1965). 
${ }^{70}$ D. ter Haar, Men of Physics: L. D. Landau, Selected Readings in Physics, Vol. 2 (Pergamon Press, 1969).

${ }^{71}$ F. Di Giacomo and E. E. Nikitin, Physics-Uspekhi 45, 515 (2005).

${ }^{72}$ C. A. Mead and D. G. Truhlar, J. Chem. Phys. 84, 1055 (1986).

${ }^{73}$ V. Betz and B. D. Goddard, SIAM J. Sci. Comput. 33, 2247 (2011). 